\title{
Redundancy Elimination of Stationary Image Using DWT
}

\author{
Prof. Dola Sanjay S. ${ }^{1}$, G. Pallavi ${ }^{2}$, B. Tarun Kumar $^{3}$, M. Tejaswini ${ }^{4}$, \\ M. Ratna Kireeti ${ }^{5}$ \\ ${ }^{I}$ Professor, Ramachandra College of Engineering, Eluru, Andhra Pradesh, India. \\ 2,3,4,5 B. Tech. Students, Department of Electronics \& Communication Engineering, Ramachandra College of \\ Engineering, Eluru, Andhra Pradesh, India.
}

\begin{abstract}
In present days, medical images and medical records are being stored in repositories so that physicians can engage in accurate diagnostic. Thus the repository requires large storage capacity and this kind of system is very expensive. So, "COMPRESSION" is one of the techniques to solve this problem. The main idea behind our paper is to compress an image, so that we can use the storage space effectively. In our project we are using lifting base DWT which is ideal for compressing images. The reason behind choosing the Lifting Base Algorithm is, complexity is reduced when compared with other algorithms and provides an efficient way to compute wavelet transform.The image compression analysis has been done by using the design metrics Peak Signal to Noise Ratio(PSNR),Mean Square Error(MSE).
\end{abstract}

Keywords: Compression, Lifting Scheme, Compression ratio, PSNR

\section{Introduction}

Uncompressed multimedia (graphics, audio and video) data requires considerably large storage capacity and transmission bandwidth. Despite rapid progress in mass-storage density, processor speeds, and digital communication system performance, demand for data storage capacity and data-transmission bandwidth continues to outstrip the capabilities of available technologies. The recent growth of data intensive multimediabased web applications have not only sustained the need for more efficient ways to encode signals and images but have made compression of such signals central to storage and communication technology. Image compression research aims at reducing the number of bits needed to represent an image by removing the spatial and spectral redundancies as much as possible. Image compression is the application of data compression on digital images. The objective is to reduce redundancy of the image data in order to store or transmit data in an efficient form. The compression in an image reduces the cost of storage and increase the speed of transmission.

There are two types of image compression techniques namely lossy and loss-less.

- The lossy type aims to reduce the bits required for storing or transmitting an image without considering the image resolution.

- The lossless type of image compression focuses on preserving the quality of the compressed image so that it is same as the original image.

Though in lossy type there is some loss of data this loss cannot be noticed by the signal receiver, namely the Human Visual System (HVS).

\section{Previous Methods:}

One of the most commonly used techniques for image compression is DCT as it has strong energy compaction property. DCT has the property that, for a typical image most of the visually significant information about an image is concentrated in just few coefficients of DCT. After the computation of DCT coefficients, they are normalized according to a quantization table with different scales provided by the JPEG standard computed by psycho visual evidence. Selection of quantization table affects the entropy and compression ratio. For the lower compression ratio, the distortion is unnoticed by human visual perception. In order to achieve higher compression it is required to apply quantization followed by scaling to the transformed coefficient. For such higher compression ratio DCT has following two limitations: (i).Blocking arti-facts (ii).False Contouring. Due to the disadvantages in DCT like blocking artifacts and false contouring we get degraded image. Hence in our proposed method we are trying to get rid of these false effects. In this paper, we are using Lifting Wavelet Transform. This transform generates an output which has better resolution when compared to previous techniques. The output image are said to be better resolute with respect to their PSNR values. The more is the PSNR value the greater is the resolution.

\section{Proposed Method:}

In this proposed method compression of an image is done using lifting base 2-D DWT. We have used lifting scheme of wavelet transform for compression because lifting scheme is having following advantages over conventional wavelet transform technique. 
- It allows a faster implementation of the wavelet transform. It requires half number of computations as compare to traditional convolution based discrete wavelet transform. This is very attractive for real time low power applications.

- The lifting scheme allows a fully in-place calculation of the wavelet transform. In other words, no auxiliary memory is needed and the original signal can be replaced with its wavelet transform.

- Lifting scheme allows us to implement reversible integer wavelet transforms. In conventional scheme it involves floating point operations, which introduces rounding errors due to floating point arithmetic. While in case of lifting scheme perfect reconstruction is possible for loss-less compression. It is easier to store and process integer numbers compared to floating point numbers.

\section{Block Diagram:}

\section{Description Of Each Block:}

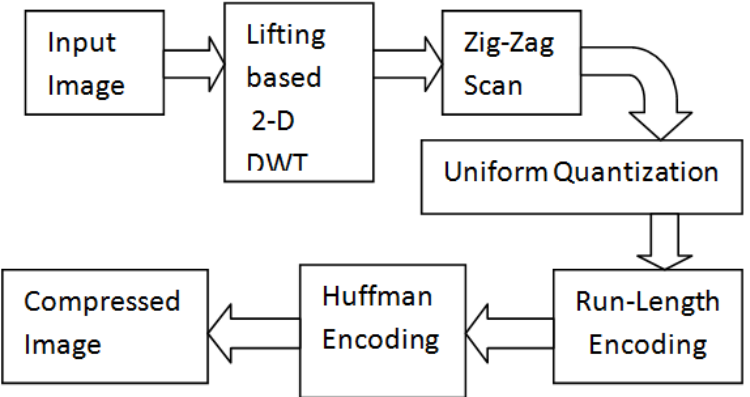

Block Diagram Of Lifting Base 2D-DWT

Input image:

Here the input is taken as the image which is to be compressed.

\section{Lifting based 2-D DWT:}

The lifting scheme is a technique for both designing wavelets and performing the discrete wavelet transform (DWT). In an implementation, it is often worthwhile to merge these steps and design the wavelet filters while performing the wavelet transform. This is then called the second generation wavelet transform. The technique was introduced by Wim Sweldens. Factorizes orthogonal and bi-orthogon al wavelet transforms into elementary spatial operators called lifting. Fortunately, a lifting step can be modified to operate on integers, while preserving the reversibility. Thus, the lifting scheme became a method to implement reversible integer wavelet transforms.

This lifting algorithm is computed at three main phases, namely:

(i) The Split phase

(ii) The Predict phase

(iii) The Update phase

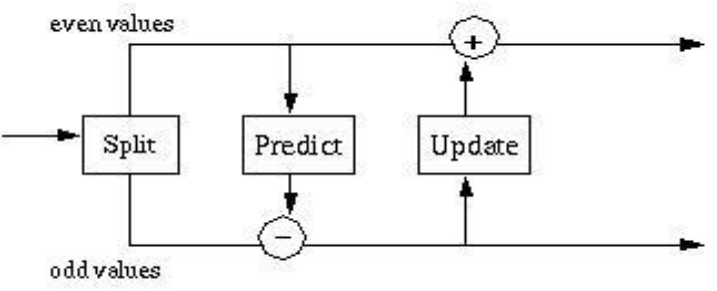

Split phase:

\section{Lifting Wavelet Scheme}

In the split phase, the data set $x(n)$ is split into two subsets to separate the even samples $x(2 n)$ from the odd ones $x(2 n+1)$.

\section{Prediction phase:}

In the prediction stage, the main step is to eliminate redundancy left and give a more compact data representation. It is proposed to use the even subset $x(2 n)$ to predict the odd subset $x(2 n+1)$ using a prediction function $\mathrm{P}$ as described in equation (3.1). The difference between the predicted value of the subset and the original value is processed and replaces the latter.

$$
\mathrm{d}=\text { Xodd }-\mathrm{P} \text { (Xeven). }
$$




\section{Update phase:}

The third stage of the lifting scheme is called the update phase. In this stage, the coefficient $\mathrm{x}(2 \mathrm{n})$ is lifted with the help of the neighboring wavelet coefficients as represented by equation (3.2). This phase is also referred as the primal lifting phase or update phase.

$$
\mathrm{s}=\text { Xeven }+\mathrm{U}(\mathrm{d}) \text {. }
$$

Where, $\mathrm{U}$ is the new update operator.

Here instead of using traditional DWT we used multi-level discrete two dimensional wavelet transform based on lifting method. It is multi-level as based on the algorithm the level can be decided on. The wavelets used are Coherent Daubechies-Feanveau (CDF) 9/7 wavelet, which has the name "cdf97". The Lifting scheme of the biorthogonal transform $9 / 7$ goes through four steps:

- Two prediction operators

- Two update operators

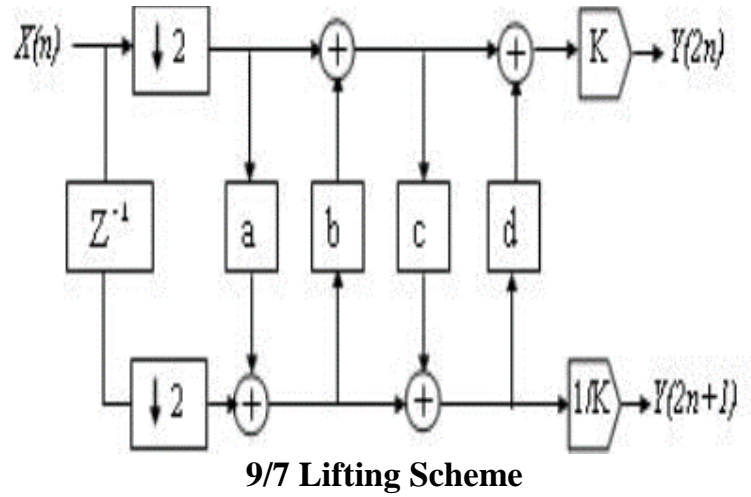

Zig - zag scan:

The most common way to scan image is one horizontal line at a time. This is also how images were drawn on displays, and how image formats like PNG, and TIFF store images. The reason to use zigzag scan is that it's reasonable to expect pixels in a block have more in common than pixels going across in a straight line. Having more in common means they can be compressed more.

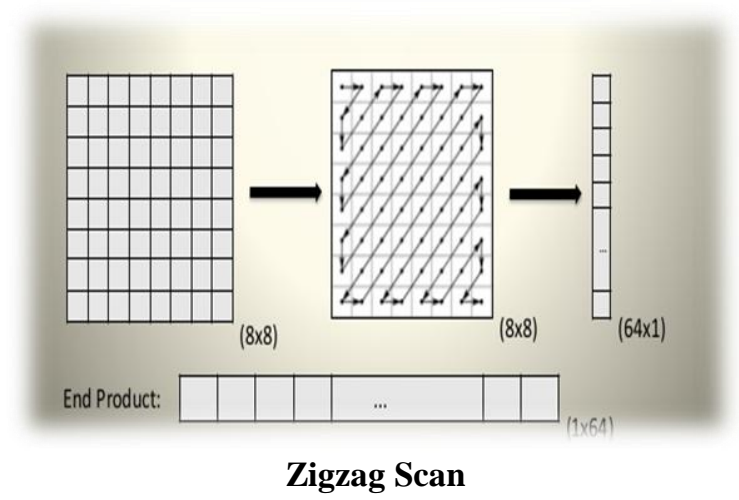

So if the block is varying shades of red and you're storing only the difference between pixels, many of the differences will hopefully be 0 or very small numbers you eliminate or make 0 . It converts $2 \mathrm{D}$ image into 1 -D image (i.e., into a single vector)

\section{Uniform quantization:}

Quantization involves compressing a range of values to a single quantum value. It involves dividing each coefficient by an integer between 1 and 255 and rounding off. 


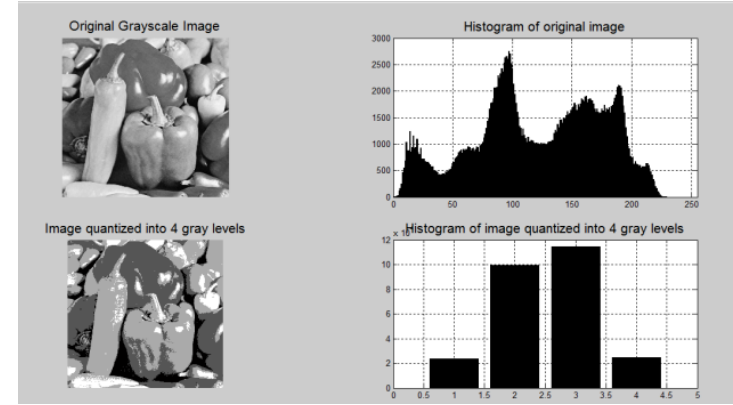

Uniform Quantization

\section{Run length encoding:}

Run-length encoding (RLE) is a very simple form of data compression in which runs of data (that is, sequences in which the same data value occurs in many consecutive data elements) are stored as a single data value and count, rather than as the original run.

\section{Huffman encoding:}

Significant level of compression can be obtained by replacing long strings of binary digits by a string of much shorter code words. A new pixel value is obtained by finding the difference between the current pixel and the predicted pixel value. The algorithm accomplishes its goals by allowing symbols to vary in length. Shorter codes are assigned to the most frequently used symbols, and longer codes to the symbols which appear less frequently in the string.Code word lengths are no longer fixed like ASCII .Code word lengths vary and will be shorter for the more frequently used characters. The Huffman code is optimal in the sense that it is the code for which the weighted path length is minimal. i.e., if $\mathrm{c}\{\mathrm{i}\}$ is the code for the $\mathrm{i}^{\text {th }}$ letter, and $\mathrm{p}(\mathrm{i})$ is the probability given to the letter, Huffman code minimizes

$$
\sum_{\mathrm{i}} \mathrm{p}(\mathrm{i}) \times \text { length }(\mathrm{c}\{\mathrm{i}\})
$$

\section{Compressed image:}

Finally after all these steps we get a compressed image.

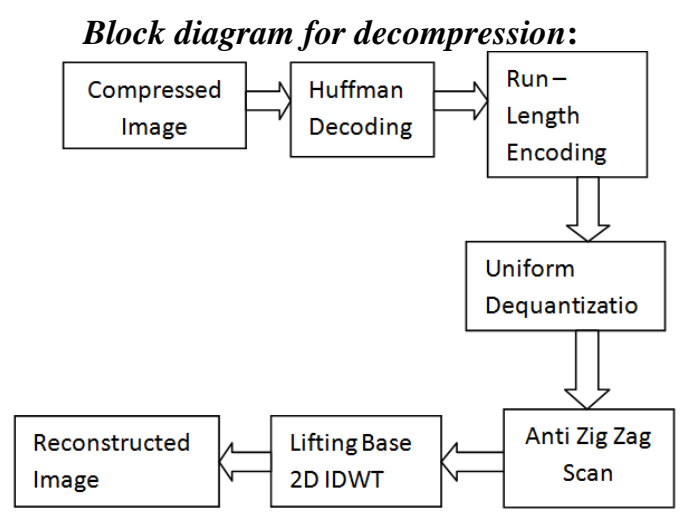

\section{Design metrics:}

Digital image compression techniques are examined with various metrics. Among those the most important one is Peak Signal to Noise Ratio (PSNR) which will express the quality. There exists another property which expresses the quality, that is, Mean Square Error (MSE).PSNR is inversely proportional to MSE. The other important metric is Compression Ratio, which express the amount of compression embedded in the technique.

\section{Compression ratio:}

It is the ratio of no. of bits required to store a original image to the no. of bits required to store a compressed image.

Where,

$$
\mathrm{Cr}=(\mathrm{n} 1 / \mathrm{n} 2)
$$

$\mathrm{n} 1-$ no. of bits required for storing an original image.

n2 -no. of bits required for storing a compressed image. 
- $\quad$ Larger compression ratio indicates more effective compression

- Smaller compression ratio indicates less effective compression

\section{Peak Signal to Noise Ratio (PSNR)}

PSNR is a measure of the signal strength.

$\mathrm{PSNR}=20 * \log 10(255 / \sqrt{\mathrm{MSE}}))$

- A higher value of PSNR is good because it means that the ratio of Signal to Noise is higher.

- Here, the 'signal' is the original image, and the 'noise' is the error in reconstruction.

- So, if you find a compression scheme having a lower MSE (and a high PSNR), you can recognize that it is a better one.

\section{Flow Chart For Compressing an image}

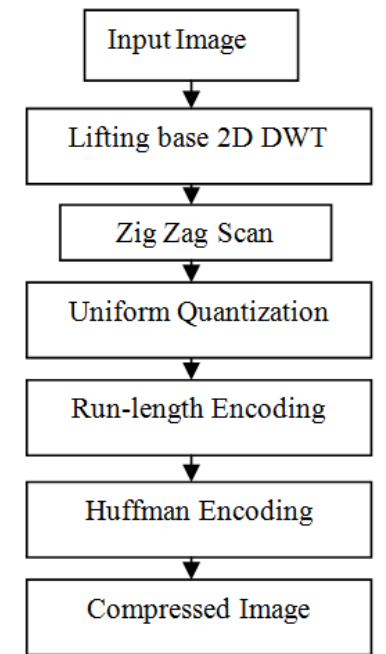

Flow Chart for De-compressing an image,

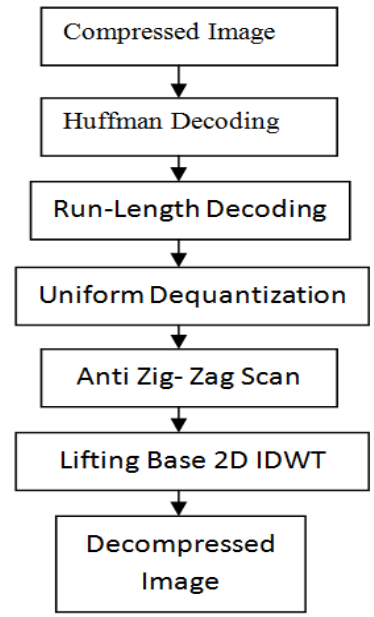

\section{Simulation Results}

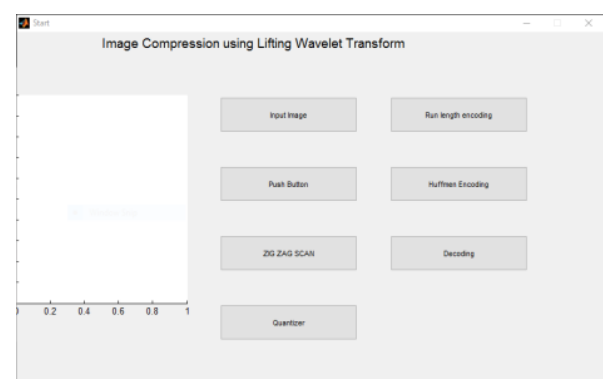

This is the GUI (Graphical User Interface) in MATLAB 


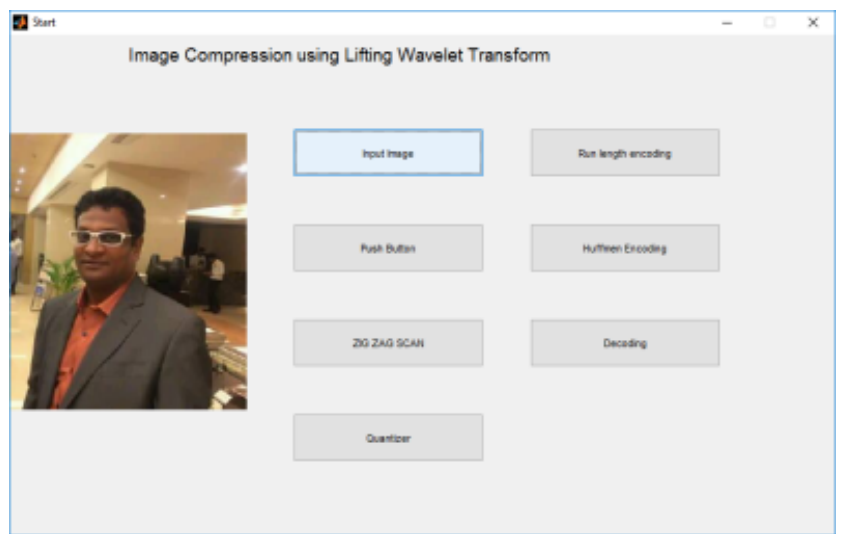

Then selected image is taken as input for processing

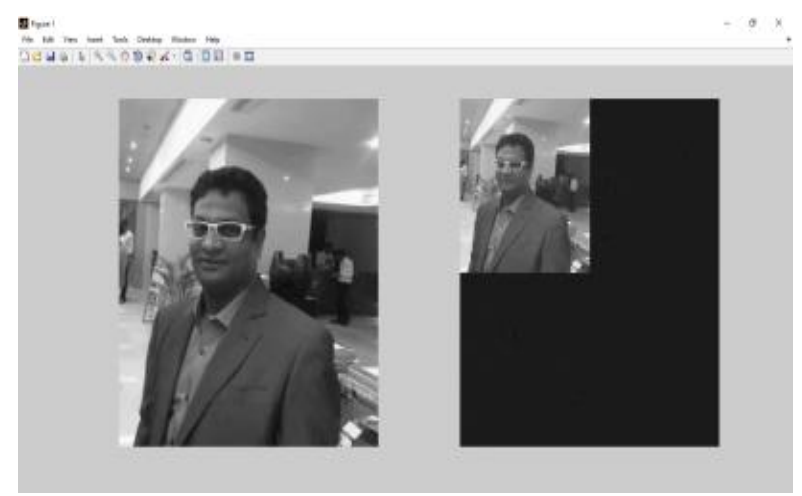

Input image after Lifting process

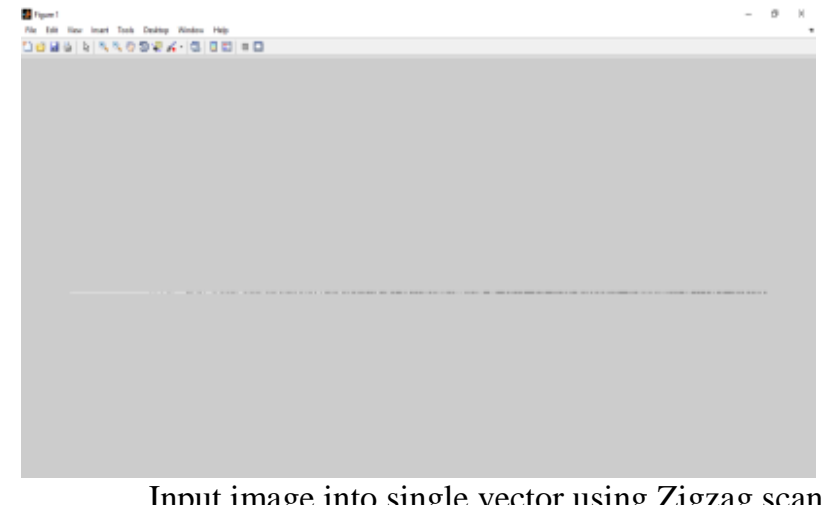

Input image into single vector using Zigzag scan

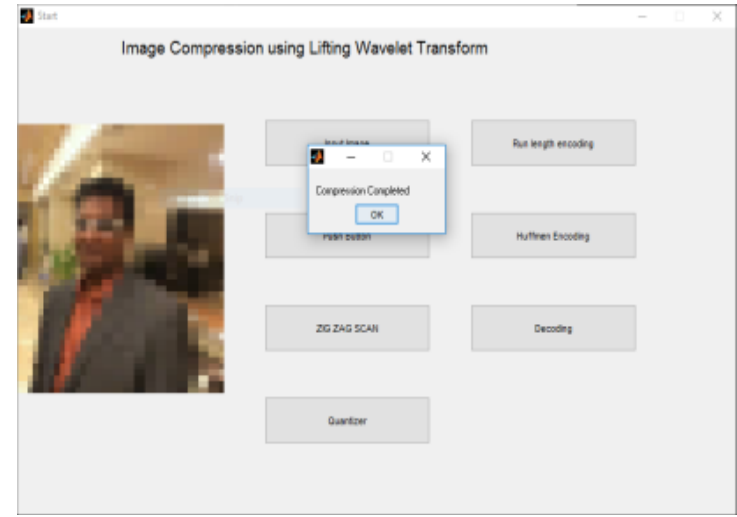

Compressed Image after Encoding Process 


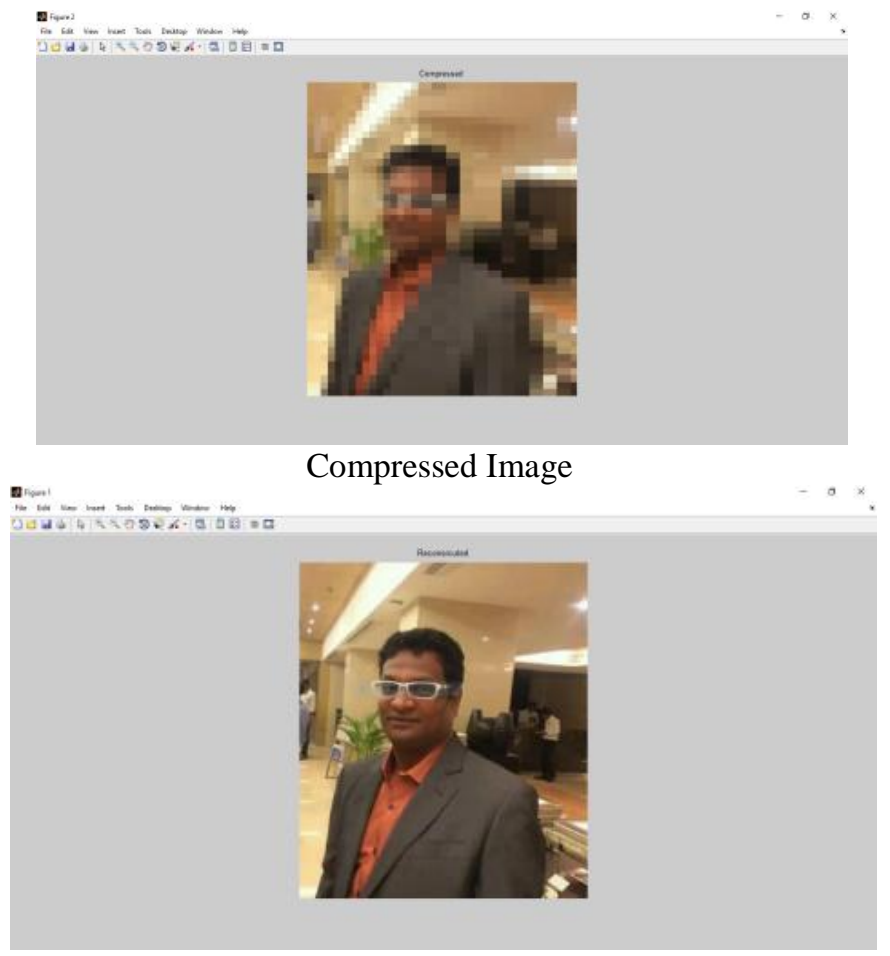

Decompressed Image

\section{Conclusion}

To conclude, we present a case of image compression using different approaches like DCT, DWT and so on. We proposed DWT technique based on lifting where the image is being compressed and while decompressing we are not affected by blocking artifacts and false countering. The reason behind satisfactory results is the use of lifting based 2-d DWT where in we are lifting the values to get the higher compression ratio and good quality image while we are decompressing. Our analytical observations on reception of compression ratio and image quality are validated by extensive MATLAB simulations.

Table: Design Metrics Table for Different Images

\begin{tabular}{|l|l|l|l|}
\hline \multicolumn{1}{|c|}{ Image } & Compression Ratio & \multicolumn{1}{c|}{ PSNR } & \multicolumn{1}{c|}{ MSE } \\
\hline Peppers & 2.5 & 34 & $4.5 \mathrm{e}^{-21}$ \\
\hline Brain MRI & 1.8 & 32 & $2.18 \mathrm{e}^{-22}$ \\
\hline Leena & 2.6 & 33 & $4.2 \mathrm{e}^{-22}$ \\
\hline Group photo & 2.5 & 33 & $1.6 \mathrm{e}^{-20}$ \\
\hline
\end{tabular}

\section{Future Scope}

As a future work, we plan to extend the current study with adaptive lifting and adaptive Huffman encoding to find a more generalized compression ratio for image and video compression applications.

\section{Reference}

[1] https://www.researchgate.net/publication/215663464_Image_Compression_using_Lifting_Wavelet_Transform

[2] Salomon, D., Data Compression, 4th Edition. Springer, 2006-07

[3] Sayood K. Introduction Data Compression, 2nd Edition 2000, Morgan Kauffmann

[4] S. Majumder, ET. al., "A Comparative Study of DCT and Wavelet-Based Image Coding \& Reconstruction”, Pg 43-46 proceedings of National Conference on Smart Communication Technologies \& Industrial Informatics, NIT Rourkela, 3-4, February 2007.

[5] S. Majumder, et. al., "Wavelet and DCT-Based Image Coding \& Reconstruction for Low Resolution Implementation" pg 814-818 International Conference on Modeling and Simulation 2007, AMSE, Dec' 07.

[6] S. Majumder, ET. al., "A comparative study of image compression techniques based on SVD, DWT-SVD and DWT-DCT" pg 500504 at International Conference on Systemic, Cybernetics,Informatics (ICSCI-2008), Hyderabad, Jan' 08

[7] http://www.mecs-press.org/ijigsp/ijigsp-v5-n11/IJIGSP-V5-N11-6.pdf

[8] http://www.slideshare.net/AishwaryaKM1/jpeg-image-compression-56894348

[9] https://www.ijedr.org/papers/IJEDR1401120.pdf

[10] http://www.ijcsi.org/papers/IJCSI-9-4-1-327-330.pdf 


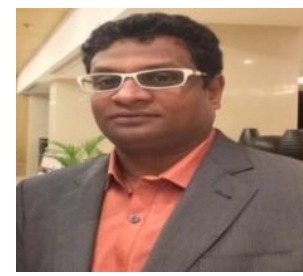

Over 15+ years experience in Teaching with reputed Engineering colleges. Currently working with VIT, Chitoor as professor and Vice Principal (Department of Electronics \& Communication Engineering). Insightful experience in teaching undergraduate and postgraduate classes in, Micro waveengineering, Antenna Wave Propagation, Field theory, Electromagnetic fields, Electromagnetic wave and Transmission lines, and related practice sessions and tutorials. Have successfully guided and implemented projects for under graduate students and post graduate students. 